\title{
Isolation and Characterization of Vaccine Candidate Genes Including CSP and MSP1 in Plasmodium yoelii
}

\author{
Seon-Hee Kim¹, Young-An Bae'2, Ju-Young Seoh ${ }^{3, \dagger}$, Hyun-Jong Yang ${ }^{1, *}$ \\ 'Department of Parasitology, Ewha Womans University School of Medicine, Seoul 07985, Korea; ${ }^{2}$ Department of Microbiology, Gachon University \\ College of Medicine, Incheon 21936, Korea; 'Department of Microbiology, Ewha Womans University School of Medicine, Seoul 07985, Korea
}

\begin{abstract}
Malaria is an infectious disease affecting humans, which is transmitted by the bite of Anopheles mosquitoes harboring sporozoites of parasitic protozoans belonging to the genus Plasmodium. Despite past achievements to control the protozoan disease, malaria still remains a significant health threat up to now. In this study, we cloned and characterized the full-unit Plasmodium yoelii genes encoding merozoite surface protein 1 (MSP1), circumsporozoite protein (CSP), and Duffy-binding protein (DBP), each of which can be applied for investigations to obtain potent protective vaccines in the rodent malaria model, due to their specific expression patterns during the parasite life cycle. Recombinant fragments corresponding to the middle and C-terminal regions of PyMSP1 and PyCSP, respectively, displayed strong reactivity against $P$. yoelii-infected mice sera. Specific native antigens invoking strong humoral immune response during the primary and secondary infections of $P$. yoelii were also abundantly detected in experimental ICR mice. The low or negligible parasitemia observed in the secondary infected mice was likely to result from the neutralizing action of the protective antibodies. Identification of these antigenic proteins might provide the necessary information and means to characterize additional vaccine candidate antigens, selected solely on their ability to produce the protective antibodies.
\end{abstract}

Key words: Plasmodium yoelii, MSP1, DBP, CSP, vaccine candidate, protective immunity, rodent malaria model

\section{INTRODUCTION}

Malaria is an infectious disease affecting humans, which is transmitted by the bite of Anopheles mosquitoes harboring sporozoites of parasitic protozoans belonging to the genus Plasmodium. Five species, including Plasmodium falciparum, $P$. vivax, P. ovale, P. malariae, and P. knowlesi, infect humans in the respective endemic areas [1,2]. The parasitic disease remains a significant health threat with more than 3 billion people at risk of infection per year. However, substantial progress has been made in fighting malaria and thus, according to the latest estimates between 2000 and 2015, case incidence and mortality rate of the protozoan disease were reduced by $41 \%$ and $62 \%$, respectively. During the period, the numbers of country, where malaria is endemic, was also decreased from 108 to 91 countries. In 2015, 212 million cases occurred globally leading to 429,000 deaths, most of which were children aged under 5

- Received 1 February 2017, revised 19 June 2017, accepted 20 June 2017.

*Corresponding author (parayang@ewha.ac.kr)

${ }^{\dagger}$ Co-corresponding author

(c) 2017, Korean Society for Parasitology and Tropical Medicine

This is an Open Access article distributed under the terms of the Creative Commons Attribution Non-Commercial License (http://creativecommons.org/licenses/by-nc/4.0) which permits unrestricted non-commercial use, distribution, and reproduction in any medium, provided the original work is properly cited. years in Africa [3].

Despites the obvious progress in antimalarial interventions, emergence and spread of drug-resistant parasites, as well as insecticide-resistant mosquitoes, are still existing risks that potentially threaten the recent gains in malaria control [4]. Approaches to develop molecules that effectively protect transmission of malaria from mosquito vector and/or generate defensive immune response in humans have been recognized as the greatest challenges in malaria elimination [5-7]. By virtues of these foresight activities, numerous Plasmodium proteins have been targeted for the development therapeutic vaccines by blocking any of the vector- or host-specific malarial stages. Some of the vaccine candidates have already entered diverse phases for clinical testing $[3,7]$.

Of the 5 Plasmodium species infecting humans, P. falciparum is the most significant cause of malaria and thus has become the major concern in the relevant areas investigating molecular tools for malaria control. However, studies on human malaria are inherently restricted by difficulty either in using humans as an experimental system or in maintaining in vitro the complete life cycle of the protozoan parasites. Rodent malaria parasites, including Plamodium berghei, P. yoelii, P. chabaudi, and P. vinckei can be a practicable choice to use as models of human 
malaria in the related experiments [8,9]. Small but significant biological difference among them in laboratory mice makes these rodent malaria particularly attractive models for the investigation of specialized aspects of human malaria. For example, $P$. berghei preferentially invades reticulocytes and induces severe pathology in mice [8]. P. chabaudi can be an appropriate model in studies associated with the drug resistance and immune evasion especially by antigenic variation [10]. In contrast, $P$. yoelii has been widely used in experiments concentrating on the liver-stage biology of malaria and on the host immune responses against that stage $[9,11,12]$. The last species is also highly applicable either in the investigations of receptors involved in erythrocyte binding [13] or in the evaluation of vaccine candidates [14-16].

In this study, we identified and cloned $P$. yoelii genes, including merozoite surface protein 1 (MSP1), circumsporozoite protein (CSP), and var 2 chondroitin sulphate A (VAR2CSA) genes, which can be applied in the investigation of immunization-induced protective immunity against $P$. yoelii malaria. The presence of antibodies specific to the recombinant fragments of MSP1 and CSP proteins was also examined in mouse sera infected with $P$. yoelii.

\section{MATERIALS AND METHODS}

\section{Experimental infections}

Male ICR mice (4-week-old) were housed in the experimental animal center of Ewha Womans University School of Medicine, Seoul, Korea under the Institutional Animal Care and Usage Committee (IACUC) approved protocol for the animal care. Parasitemia of the non-lethal 17XNL strain of P. yoelii (courtesy of Prof. ET Han, Kangwon National University, Chuncheon, Korea) was monitored by microscopic examination of Diff-Quik stained blood smears from passage mice and expressed as the percentage of parasitized RBCs. Blood-stage infection was initiated by intraperitoneal injection of $150 \mu \mathrm{l}$ of the parasitized erythrocytes (5.5\%). From day 2 post challenge, the resulting parasitemia was periodically monitored by enumerating parasitized erythrocytes in thin tail-blood smears stained with Giemsa, as previously described [17]. A total of 6 experimental mice that were recovered to normal levels 3 weeks after the primary injection were reinfected with the $P$. yoelii cells (5.5\%) at 4 weeks after the first infection. Changes in the ear color and parasitemia were similarly checked in the experimental group.

\section{Parasite materials}

Blood was collected through cardiac puncture from experimental mice $(n=6)$ at day 10 after the primary and secondary infections of $P$. yoelii 17XNL strain. Plasma samples prepared from the blood were used as the 1st and 2nd antisera, respectively, specific to P. yoelii antigens. Blood was also collected from uninfected normal mice with the same age $(n=6)$ to prepare the normal control sera. The sera were stored at $-80^{\circ} \mathrm{C}$ until use.

The trophozoite and schizont stage parasites in infected mice blood from the primary and secondary infections (parasitemia $>35 \%$ ) were highly enriched by the Optiprep (iodixanol; Axis-shield, Oslo, Norway) density gradient, as previously described [18]. Cellular fraction of normal mice blood was also prepared to use as control mouse proteins. Proteins were extracted by incubating the cellular fraction samples in a protein lysis buffer $\left(155 \mathrm{mM} \mathrm{NH}_{4} \mathrm{Cl}, 12 \mathrm{mM} \mathrm{NaHCO}_{3}\right.$ and 0.1 mM EDTA) containing 1\% SDS and protease inhibitors (Roche, Mannheim, Germany) for $1 \mathrm{hr}$ on ice. The solutions were centrifuged at $12,000 \mathrm{rpm}$ for $10 \mathrm{~min}$ at $4^{\circ} \mathrm{C}$ and the centrifugal supernatants were taken as protein samples. The total protein concentrations were determined by the Lowry method and stored at $-80^{\circ} \mathrm{C}$ until use.

Genomic DNA of P. yoelii 17XNL was extracted from whole blood collected from infected mice (parasitemia $>35 \%$ ) using the QIAamp DNA Blood Mini Kit according to the manufacturer's instruction (Qiagen, Hilden, Germany). The concentration and quality of the prepared genomic DNA was determined by a spectrophotometer by measuring absorbance at 260 and $280 \mathrm{~nm}$. The DNA sample was stored at $4^{\circ} \mathrm{C}$ until use.

\section{Identification of $P$. yoelii genes}

As vaccine candidates applicable in the examination of protective immunity, 4 malaria proteins (MSP1, CSP, VAR2CSA, and liver stage antigen 1 [LSA1]) were selected in this study. P. yoelii genes homologous to the selected molecules were searched in the genomic database of the rodent malaria in PlasmoDB (http://plasmodb.org/plasmo/) using BLAST algorithms. Protein and mRNA sequences used as queries in the homology searches were retrieved from the GenBank (https:// www.ncbi.nlm.nih.gov/) database of Plasmodium spp. (no. AAA66185.1 for MSP1, partial sequence of $P$. yoelii protein; no. AAY34563.1 for CSP, P. yoelii; no. ALB01043.1 for VAR2CSA, P. falciparum; and no. CD064792.1 for LSA1, P. reichenowi). The genomic nucleotide sequences were compared to the respec- 
tive mRNA sequences and then, their genomic structures were determined by referencing the general GT-AG rule [19].

\section{Amplification of full-unit genes}

Forward and reverse primers were designed to match the $5^{\prime}$ and $3^{\prime}$-ends of $P$. yoelii genes. The primer sequences were as followings: 5'-ATGAAGAAGTGTACCATTTTAGTTGTAGCGTC-3' and 5'-TTAATTAAAGAATACTAATACTAATAATATTAC-3' for CSP gene; 5'-ATGAAGGTGATTGGACTTTTATTTTCTTTC G-3' and 5'-TTAAAATATATTAAATACAATTAATGTGATAA-3' for MSP1 gene; 5'- ATGAACAAAATAATAACTTATTTTTCTTTTATAC- 3 ' and 5' - TTATTTTAAAAATATGACTATGCATACCATC-3' for VAR2CSA gene. The primers were used in long-range PCR to amplify each of the full-length genes from the genomic DNA of P. yoelii. The typical 2-step thermal cycling PCR was performed for the amplification using the LA Taq polymerase (Takara, Shiga, Japan) (initial denaturation at $95^{\circ} \mathrm{C}$ for $5 \mathrm{~min}$, 35 cycles of $45 \mathrm{~s}$ at $94^{\circ} \mathrm{C}$ and $5 \mathrm{~min}$ at $64^{\circ} \mathrm{C}$, and a final extension at $70^{\circ} \mathrm{C}$ for $10 \mathrm{~min}$ ). After verifying their correct sizes and purity on 0.8 -1\%-agarose gels, the products were cloned into a pGEM-T Easy vector (Promega, Madison, Wisconsin, USA). The nucleotide sequences were automatically determined using a BigDye Terminators Cycle Sequencing Core Kit (Ver. 3 · ; Perkin Elmer, Foster City, California, USA) and an automated ABI PRISM 377A DNA Sequencer (Applied Biosystems, Foster City, California, USA).

\section{Sequence analysis}

Open reading frame (ORF) encoded in each of the cloned genes was predicted using ORF Finder (http://www.ncbi.nlm. nih.gov/) and their similarity profiles were further verified using BLASTp with the theoretically translated amino acid (aa) sequences. The aa sequences of orthologous proteins identified in the PlasmoDB and GenBank databases were aligned with ClustalX and used in phylogenetic analysis with MEGA 6.0 (neighbor-joining algorithm, Jones-Thornton-Tayler model for aa substitution, rate heterogeneity with 4 gamma category, pairwise-deletion of gapped region sequences). The statistical reliability of branching nodes was assessed by the standard bootstrapping test of 1,000 replicates. The aa sequences were also applied in the prediction of functional protein domains using the SMART program (http://smart.embl.de/).

\section{Generation of recombinant MSP1 and CSP proteins}

The coding DNA sequences of MSP1 and CSP genes were segmentally amplified from the genomic DNA of $P$. yoelii with specific primers containing restriction sites for endonucleases (Supplementary Table 1). After digestion with the corresponding enzymes, the amplicons were cloned into pET-28a or pGEX-6p-1 plasmid and transformed into competent Escherichia coli BL21 (DE3) cells. Expression of these recombinant proteins were induced by incubating the cells in Luria-Bertani medium supplemented with $0.5 \mathrm{mM}$ isopropyl- $\beta$-Dthiogalactopyranoside (IPTG) for $4 \mathrm{hr}$ at $37^{\circ} \mathrm{C}$. The proteins were purified by nickel-nitrilotriacetic acid (Qiagen) or glutathione sepharose (GE Healthcare, Little Chalfont, UK) chromatography according to the manufacturers' instructions.

\section{Western blot analysis}

The cellular proteins (each $20 \mu \mathrm{g}$ ) extracted from the bloods of normal and infected mice were resolved through $10 \%$ SDSPAGE, together with a pooled normal mice serum $(n=5)$. The protein bands were either visualized by staining with Coomassie brilliant blue R-250 or transferred electrophoretically onto nitrocellulose membranes. The membranes were reacted with the specific mouse antisera prepared during the primary and secondary infections of $P$. yoelii $17 \mathrm{XNL}$, as well as normal mouse serum (1:1,000 dilution). Antigen-specific antibodies were detected with the goat anti-mouse IgG antibody conjugated with IR-Dye 800cw (1:20,000 dilution). The positive reaction was scanned on an Odyssey CLx imager (800 channel; Lincoln, Nebraska, USA). Images were acquired on the CLx imager at $169 \mu \mathrm{m}$ resolution. The recombinant MSP1 and CSP fragments (each $200 \mathrm{ng}$ ) were similarly applied in the western blot analysis.

\section{RESULTS}

\section{P. yoelii MSP1 gene (PyMSP1)}

A partial protein sequence (459 aa, 2104291A) corresponded to the N-terminal portion of PyMSP1 was identified in the GenBank database [20]. The sequence was used as a query during the tBLASTn analysis of $P$. yoelii transcripts in PlasmoDB to isolate the full PyMSP1 gene sequence. A total of two 5,319-bp transcripts (sequence identity $=100 \%$ ) were detected in the similarity analysis (PY05748 of $P$. yoelii yoelii 17XNL and PYYM_0834100 of P. yoelii yoelii YM). By using the sequence as a query in BLASTn search, the full-length sequence of a single exonic PyMSP1 gene was identified in the genomic databases of $P$. yoelii 17XNL/17X and P. yoelii YM strains (se- 
quence identity $=100 \%$ ). The full gene sequence was amplified by a long-range PCR from the genomic DNA of P. yoelii 17XNL (Supplementary Fig. 1).

The 1772-aa PyMSP1 protein detected orthologous proteins in almost all of the Plasmodium databases in PlasmoDB, except for that of an avian malaria P. gallinaceum, and exhibited identity values ranging from $32 \%$ (P. falciparum MSP1) to $74 \%$ ( $P$. berghei MSP1) with its plasmodial orthologs. As previously shown during characterization of P. berghei MSP1 [21], PyMSP1 also contained the structural/functional domains conserved in the plasmodial MSP1 proteins (data not shown). In a phylogenetic analysis, plasmodial MSP1 proteins showed a clustering pattern identical to that of their donor organisms [22]. The distance values were similarly estimated as $0.403 \pm$ 0.1028 and $0.408 \pm 0.5110$ among MSP1 proteins identified in rodent and primate malaria, respectively. However, the value was greatly increased to $1.535 \pm 0.0398$ between rodent and primate malaria proteins (Fig. 1).

\section{P. yoelii CSP gene (PyCSP)}

The genomic sequence of PyCSP gene was searched in PlasmoDB using BLAST programs with the mRNA (1284 bp, DQ012939.1) and aa (427 aa, AAY34563.1) sequences of $P$. yoelii CSP, which had been isolated from GenBank. The gene

A

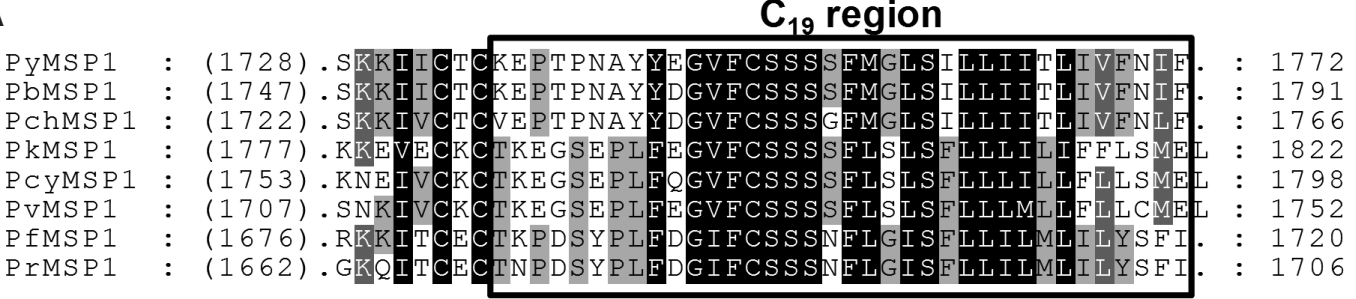

B

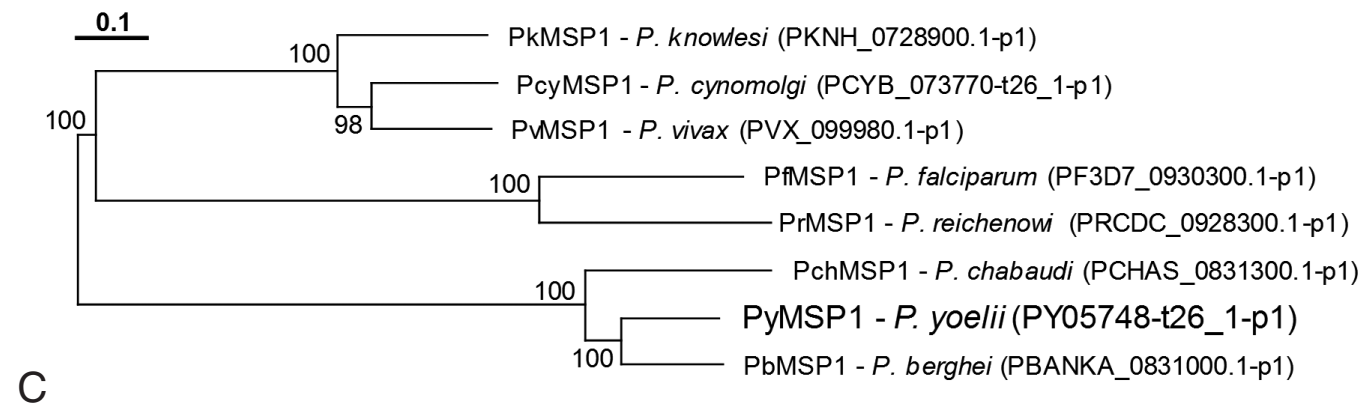

\begin{tabular}{|c|c|c|c|c|c|c|c|c|}
\hline & 1 & 2 & 3 & 4 & 5 & 6 & 7 & 8 \\
\hline 1. PyMSP1 & & $0.29 \pm 0.014$ & $0.47 \pm 0.021$ & $1.49 \pm 0.060$ & $1.52 \pm 0.067$ & $1.51 \pm 0.061$ & $1.88 \pm 0.080$ & $1.87 \pm 0.082$ \\
\hline 2. PbMSP1 & & & $0.45 \pm 0.021$ & $1.48 \pm 0.061$ & $1.54 \pm 0.068$ & $1.53 \pm 0.063$ & $1.87 \pm 0.078$ & $1.91 \pm 0.083$ \\
\hline 3. PchMSP1 & & & & $1.57 \pm 0.062$ & $1.57 \pm 0.067$ & $1.60 \pm 0.062$ & $1.96 \pm 0.084$ & $1.98 \pm 0.084$ \\
\hline 4. PkMSP1 & & & & & $0.43 \pm 0.019$ & $0.45 \pm 0.020$ & $1.46 \pm 0.062$ & $1.58 \pm 0.069$ \\
\hline 5. PcyMSP1 & & & & & & $0.35 \pm 0.015$ & $1.52 \pm 0.064$ & $1.57 \pm 0.069$ \\
\hline 6. PvMSP1 & & & & & & & $1.45 \pm 0.062$ & $1.49 \pm 0.069$ \\
\hline 7. PfMSP1 & & & & & & & & $0.62 \pm 0.028$ \\
\hline 8. PrMSP1 & & & & & & & & \\
\hline
\end{tabular}

Fig. 1. Comparison of primary structures among merozoite surface protein 1 (MSP1) identified in various Plasmodium spp. (A) The full amino acid sequence of $P$. yoelii MSP1 (PyMSP1) was aligned with those of orthologous proteins identified in other Plasmodium spp. Dots in the alignment were introduced to increase identity values among them. Only the region corresponding to the $B$ cell-activating epitopes (C19 region) is presented in this panel. (B) Phylogenetic relationships among the plasmodial MSP1 proteins were analyzed with the neighbor-joining algorithm of MEGA. All the sequences used in the analysis were retrieved from PlasmoDB (their respective entry numbers are presented in the parentheses). Numerals at branch nodes indicate their percentage of appearances in 1,000 bootstrap replicates. (C) The pairwise distance among the plasmodial MSP1 proteins was calculated with the MEGA program. 
was also found to be single exonic. However, in contrast to $M S P 1$, there were several gaps with various lengths in the resulting sequence alignment between the mRNA query and matched genomic sequences. Gap lengths in the alignment were slightly different among genomic sequences of $P$. yoelii strains, mainly due to the nucleotide length polymorphism in the repetitive region detected in the middle region of rodent malaria CSP genes [23]. The full-unit PyCSP gene empirically determined (PyCSP_17XNL-emp; Supplementary Fig. 1), had a size identical to that of DQ012939.1, although 7 nucleobase mismatches were observed between them ( $99.5 \%$ identity). Of the 7 mismatched bases, 5 bases changed the decoding amino acids (see PyCSP_17XNL-emp and AAY34563.1 in Fig. 2).

To verify the length polymorphism among the PyCSP proteins of different $P$. yoelii strains, orthologous sequences were isolated from PlasmoDB and GenBank. Two unique sequences were identified in 17XNL (PY07368-t26_1-p1/XP_728216.1 and AAY34563.1) and 17X (PY17X_0405400.1-p1/CDZ 10959. 1 and AAA29558.1) strains, whereas only a single unique sequence was detected in the proteomic database of YM strain (PYYM_0405600.1-p1/CDU16435.1). Sequence alignment of these PyCSP proteins together with that of $P$. berghei (PbCSP) demonstrated that the length polymorphism was induced by shrinkage/expansion of the repeat unit 'QGPGAP' found in the middle region of PyCSP (Fig. 2). It was also apparent that the repeat-region sequences, but not the $5^{\prime}$ - and 3 '-flanking regions, has been specifically evolved in each of the rodent malaria species. CSP proteins identified in primate malaria also exhibited repeat sequences specific to the lineages (data not shown). Amino acid sequence acting as the cytotoxic $\mathrm{T}$ lymphocyte epitope was tightly conserved in the PyCSP proteins with different strain origins (double underlined in Fig. 2) [24].

\section{VAR2CSA and LSA1 genes in Plasmodium spp.}

The VAR2CSA gene was screened in the genome of various $P$. yoelii stains by tBLASTn program using the aa sequence of $P$. falciparum VAR2CSA (partial, ALB01043.1) as a query. The sequence retrieved an mRNA transcript putatively encoding Duffy-binding protein (DBP; PY04764 of 17XNL strain, PY17X_1337400 of 17X strain and PYYM_1334400 of YM strain; $E$-value $=7 \mathrm{e}-13)$. The 5'- and 3'-region sequences of PY04764 (2361 bp) differed from those of PY17X_1337400

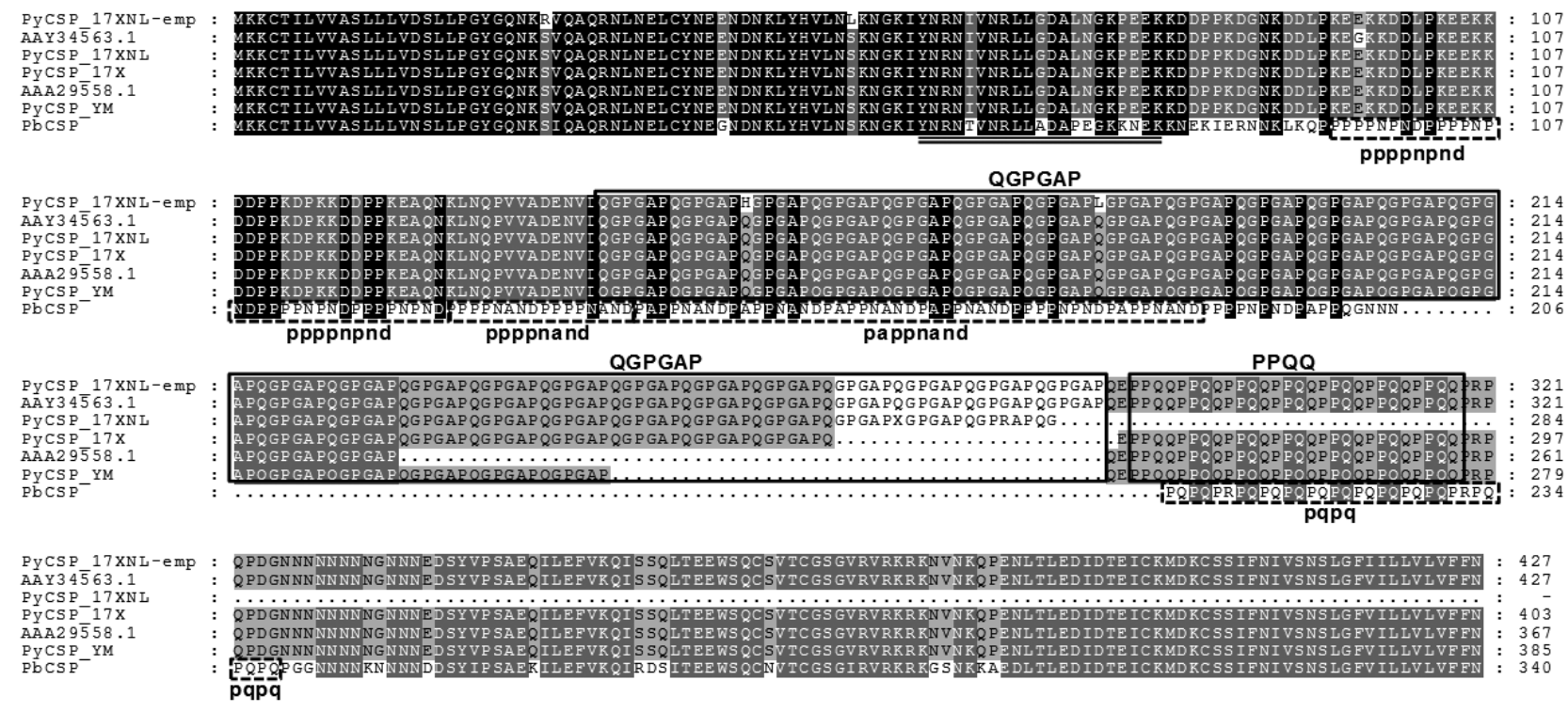

Fig. 2. Polymorphic repeat sequences of circumsporozoite proteins (PyCSP) encoded in the different Plasmodium yoelii strains. The amino acid sequence of PyCSP protein was empirically determined from the non-lethal 17XNL (PyCSP_17XNL-emp) strain in this study and compared with those identified in the PlasmoDB and GenBank databases of 17XNL (PyCSP_17XNL and AAY34563.1), 17X (PyCSP_17X and AAA29558.1), and YM (PyCSP_YM) strains. Sequence of an orthologous protein in P. berghei (PbCSP) was also included in the comparison. Dots in the alignment were introduced to increase identity values among them. Boxes with solid lines indicate repeat units conserved in the central region of CSPs of the multiple $P$. yoelii strains, while those with dotted lines mark the repeats detected in the P. berghei CSP protein. The amino acid stretches that are involved in the activation of cytotoxic T cells are double underlined. 
and PYYM_1334400 (2559 bp; data not shown). The nucleotide sequence of homologous gene, which was empirically determined from the genome of 17XNL strain (Supplementary Fig. 1), was found to be identical to those of PY17X_1337400 and PYYM_1334400. Therefore, there might have been some fault during determination of PY04764 sequence. The genomic version of the P. yoelii DBP gene ( $P y D B P, 3,228 \mathrm{bp}$ ) was comprised of 5 exons and 4 intervening introns.

BLASTp search of the GenBank plasmodial databases using the PyDBP sequence retrieved numerous DBPs from the rodent and primate malaria databases including $P$. falciparum DBP (SBT76215.1) (identity $>33 \%$, E-value $<2 e-43$ ). However, the orthologs of $P$. falciparum VAR2CSA, named erythrocyte membrane protein 1 (EMP1), were detected only in the proteome databases of $P$. reichenowi and $P$. gaboni (identity $>35 \%$, $E$-value $<6 e-23)$. The DBP and EMP1/VAR2CSA protein groups possessed the well conserved Duffy-binding domain, although the domain was duplicated in the EMP1/VAR2CSA (Fig. 3A). P. reichenowi EMP1 (XP_012760428.1) had 4 different Duffy- binding domains. Other group-specific domains such as EBA175_VI and NTS-2 were further found in the proteins of each group. In a phylogenetic tree, the Duffy-binding domains comprising DBP formed a tight clade, which were clearly separated from those consisting of EMP1/VAR2CSA (Fig.3B).

The plasmodial orthologs of LSA1 were screened with the amino acid sequence of $P$. reichenowi LSA1 (CD064792.1) against Plasmodium databases in PlasmoDB. However, matches with statistical significance were detected only in $P$. falciparum (PF3D7_1036400.1-p1 of 3D7 strain and PFIT_1035600.1-p1 of IT strain; E-value<7e-155). Therefore, LSA1 gene appeared to have evolved specifically in the genomes of $P$. falciparum and $P$. reichenowi.

\section{Protective immune responses in $P$. yoelii 17XNL- challenged mice}

The ICR mice $(\mathrm{n}=6)$ were challenged with the non-lethal $P$. yoelii 17XNL and monitored over the course of infection for parasitemia (Fig. 4). The percent parasitemia in the peripheral

A

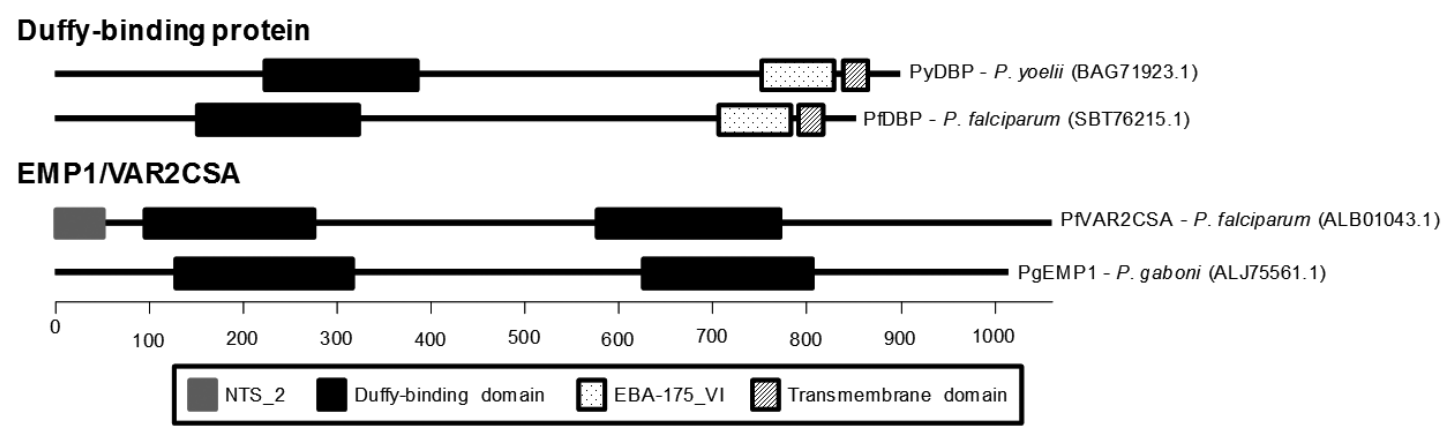

B

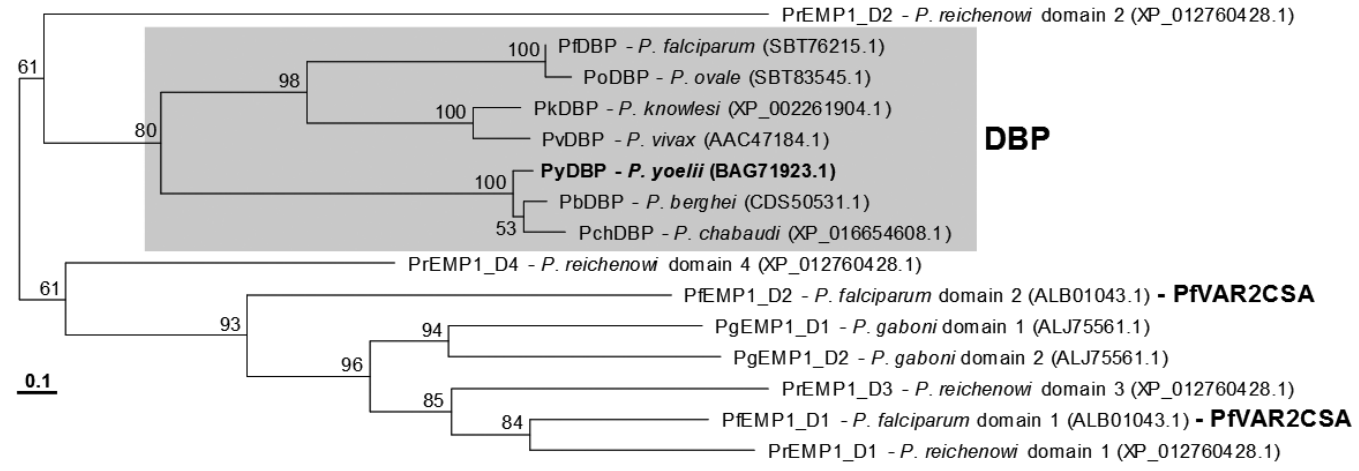

Fig. 3. Structural comparison between Duffy-binding proteins (DBP) and erythrocyte membrane protein 1 (EMP1)NAR2CSA. (A) Functional domains conserved in the representative DBP and EMP1NAR2CSA were predicted using the SMART program. (B) A neighborjoining tree was constructed with MEGA using the Duffy-binding domain sequences conserved in DBP and EMP1NAR2CSA proteins of Plasmodium spp. Multiple Duffy-binding domains encoded in EMP1NAR2CSA were marked as D1, D2, and so on. Numerals at branch nodes indicate their percentage of appearances in 1,000 bootstrap replicates. Proteins annotated as DBP are highlighted in grey box. 
blood was increased from $2.03 \% \pm 0.403$ at 2 days to $43.82 \% \pm 15.146$ at day 10 post-infection. The peak parasitemia at day 10 was gradually decreased to a background level and finally, complete convalescence from malaria was confirmed at week 3 after the primary challenge. The mice ears, which had turned to be pale during the infection with high parasitemia, recovered their original color (Fig. 4B). Interestingly, the parasite was rarely detected in the peripheral blood, when the recovered mice were secondary challenged with an identical dose of $P$. yoelii 17XNL ( $n=6$; Fig. 4A-C). The mice ears also maintained the normal pinkish color (Fig. 4B). Western blot analysis of $P$. yoelii proteins with a pooled serum collected from the primary and secondary infected mice $(n=6)$ demonstrated that multiple proteins invoked humoral immune response to generate specific antibodies (Fig. 4D). Some of the specific antigen-antibody reactions were uniquely de-

A

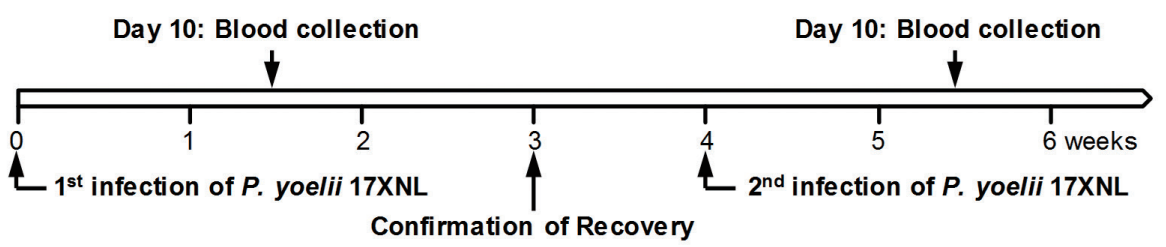

B

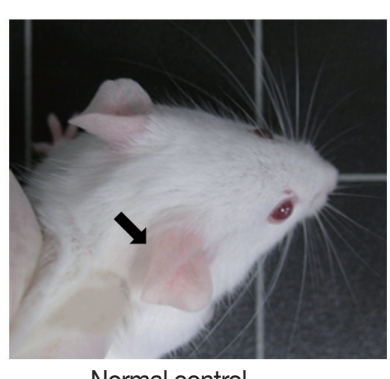

Normal control

C

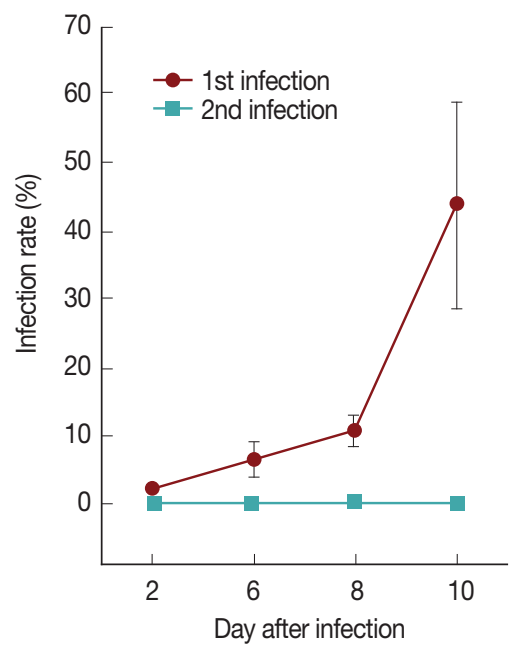

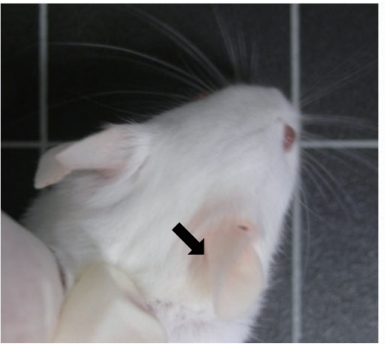

1st infection

$\mathrm{D}$

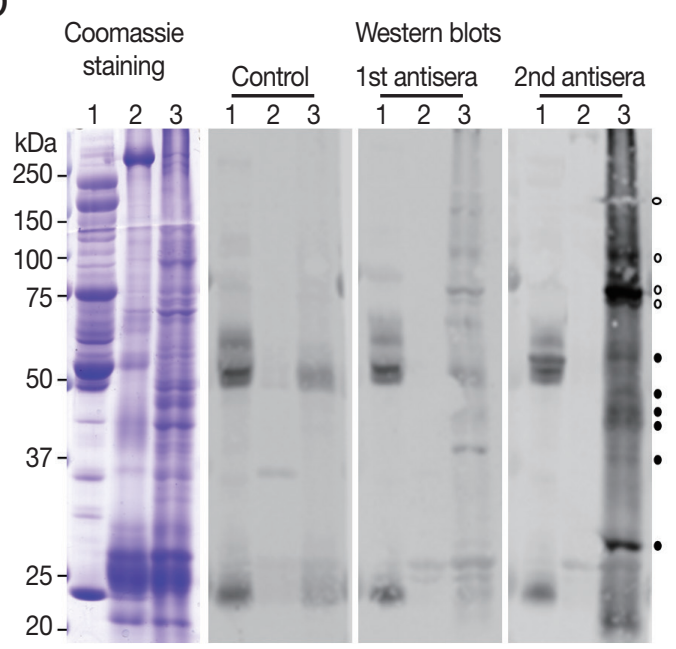

Fig. 4. Infection of the non-lethal Plasmodium yoelii 17XNL strain to experimental ICR mice. (A) Schematic presentation of time schedule for the primary and secondary injection of the parasite into the mice and for blood collection to prepare specific antisera and parasitic materials. (B) Observation of ear color during the primary and secondary infections (black arrows). The images were taken from the experimental- (1st and 2nd infections) and control-group mice at 10 days post-infection. (C) Monitoring of parasitemia by enumerating parasitized RBCs in thin tail-blood smears stained with Giemsa. Mean \pm SD. (D) Detection of $P$. yoelii proteins-specific antibodies in the mice antisera by Western blot analysis. The sera were collected from the primary- (1st antisera) and secondary (2nd antisera)-infected mice as well as normal mice (control). Lane 1, plasma proteins in normal mice blood; lane 2, proteins extracted from cellular portion of normal mice blood; lane 3, proteins extracted from cellular portion of the primary-infected mice blood at 10 days post-infection. Closed circles mark protein bands reacted only with the 2nd antisera, while open circles indicate protein bands, of which positive signal was stronger with the 2nd antisera than that with the 1st antisera. 
A

PyMSP1

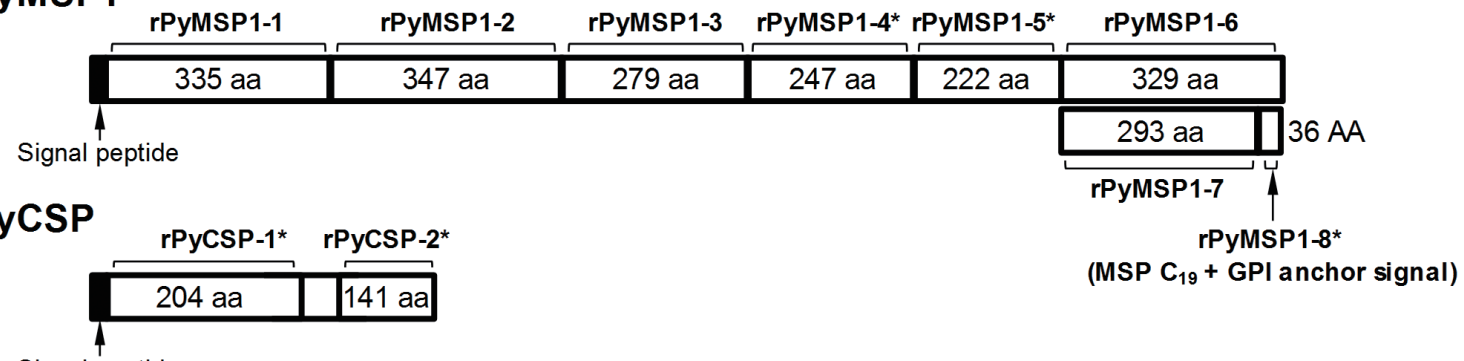

Signal peptide

B

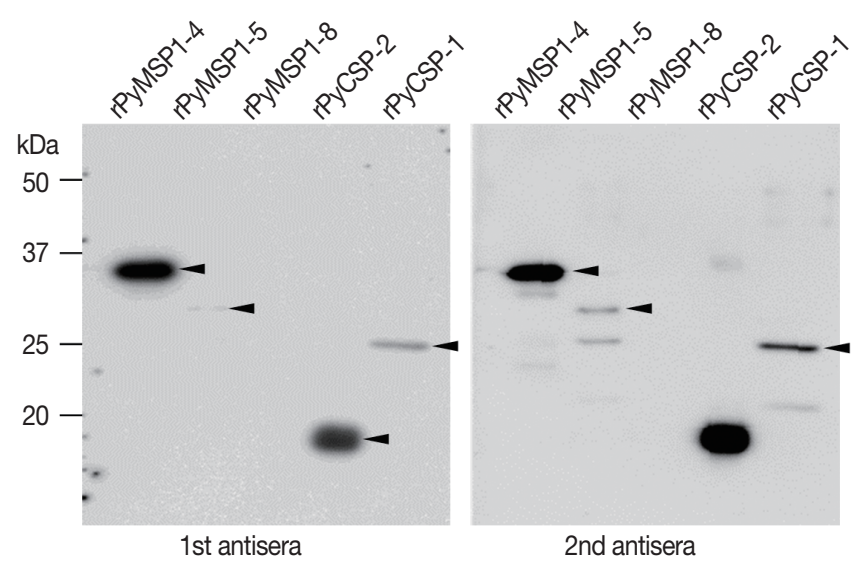

Fig. 5. Antigenicity of recombinant fragments of Plasmodium yoelii MSP1 and CSP proteins. (A) Schematic representation of cloned sites for the generation of each recombinant fragment. Asterisks mark proteins, which had been successfully induced in this study. (B) Detection of the recombinant protein-specific antibodies in mouse antisera collected from the first and second infections. Arrowheads indicate the positions of positive signals.

tected in the secondary infected serum (closed circles), while the others were found to be intensified probably due to the repeated challenge of identical antigens in the re-infected mice (open circles).

\section{Antigenicity of recombinant MSP1 and CSP proteins}

The coding DNA sequences of $P$. yoelii MSP1 and CSP genes were segmentally cloned to generate recombinant fragments of these proteins (Fig. $5 \mathrm{~A}$ and Supplementary table 1). Of the 10 gene segments transformed into E. coli cells, 5 gene segments were successfully induced by IPTG ( 3 rPyMSP1 and 2 rPyCSP segments; recombinant fragments marked with asterisks). The recombinant proteins were purified and reacted with the P. yoelii-specific mouse antisera. As shown in Fig. 5B, the rPyMSP1-4 and rPyCSP-2 fragments exhibited strong positive signal, while rPyMSP1-5 and rPyCSP-1 reacted weakly with the mouse sera. All the signal intensity was slightly increased in reactions with sera from the secondary infection.
However, no significant reaction was observable between the mouse antisera and rPyMSP1-8 containing $\mathrm{C}_{19}$ fragment, which had been known to act as a potent B-cell epitope in $P$. falciparum MSP1.

\section{DISCUSSION}

Plasmodial proteins that are specifically expressed during erythrocyte stage have been targeted for the development of protective vaccines $[7,25,26]$. Up to date, several proteins expressed on the surface of the merozoites and infected red blood cells such as merozoite surface proteins $1 / 2 / 3$ (MSP1/ $2 / 3$ ), serine-repeat antigen, erythrocyte binding antigen, glutamate-rich protein (GLURP) and apical membrane antigen 1(AMA1) have been developed as potent vaccines [7]. MSP1 engaging in the initial attachment of merozoites to erythrocytes is a leading erythrocyte-stage vaccine candidate, which has been most extensively studied in the relevant fields [27]. 
Both of the N-terminal and C-terminal fragments of MSP1 have been found to be effective for the generation of immune response and protection against malaria [28,29], although the C-terminal fragment appeared to exhibit larger effect than the $\mathrm{N}$-terminal counterpart [26]. The protective immunity has also been examined with rodent model using the C-terminal fragment of MSP1 protein expressed in P. yoelii [16,30]. However, it was unclear whether the C-terminal fragment of native PyMSP1 invoke generation of specific antibody during P. yoelii infection, since no significant immune reaction was detected against the C-terminal $\mathrm{C}_{19}$-containg fragment of PyMSP1 (rPyMSP1-8; Fig. 5). In addition, the middle fragment of PyMSP1 A (rPyMSP1-4) also exhibited strong reactivity against mouse sera infected with the parasite.

CSP is a sporozoite stage-specific protein that expresses a unique immunodominant epitope recognized by immunized or repeatedly infected hosts $[24,31,32]$. The plasmodial protein contains $\mathrm{N}$ - and $\mathrm{C}$ - terminal regions of relatively low immunogenicity, which flank a central region with tandemly repeated aa units. The central repeats differ from species to species and have been targeted as vaccine candidates for protective immunity against malarial infection due to their highly immunogenic properties $[23,33,34]$. The central repeats of CSP encoded in the P. yoelii genome were 'QGPGAP' and 'PPQQ', which were separated by a di-amino acid sequence ' $\mathrm{QE}$ '. The repeat units were well-conserved in the CSP orthologs identified in different $P$. yoelii strains (Fig. 2). The significant length polymorphism observed among these orthologs were mainly resulted from the shrinkage/expansion of the former 'QGPGAP' repeat. Meanwhile, the repeats were 'PPPPNPND' and 'PQPQ' in CSP protein of $P$. berghei, while the sequence of former unit was slightly deviated to 'PPPPNAND' and 'PAPPNAND' in the downstream regions. More recent investigations demonstrated that the N-terminal region of CSP proteins (double underlined sequence in Fig. 2) can also be applicable for the development of protective vaccines, even though its protective potential was relatively weak compared to that of the central repeat $[24,34]$. In P. yoelii system, the specific mouse antisera reacted strongly with the $\mathrm{C}$-terminal fragment of CSP (rPyCSP-2), but not with the N-terminal fragment containing the 'QGPGAP' repeat (rPyCSP-1) (Fig. 5B). Taken together, these facts may reflect the collaborated actions between multiple regions of CSP proteins, which invoke specific immune response(s) against the parasite infection, are uniquely evolved among Plasmodium species.
The adhesion of infected erythrocytes to vascular endothelium or placenta is the key event in the pathogenesis of severe malaria induced by $P$. falciparum infection. By binding to receptors in the vascular bed, the parasite avoids being filtered through spleen, where they are removed from the circulation $[35,36]$. The plasmodial EMP1 proteins are adhesion molecules mediating the interaction between infected erythrocytes and the host endothelium. Of the multiple paralogous EMP1 proteins, VAR2CSA is a unique variant of EMP1 that is expressed in pregnant women and is responsible for the adhesive binding in the placenta. The VAR2CSA protein interacts with a low-sulfated form of chondroitin sulfate $\mathrm{A}$ attached to proteoglycans in the intervillous spaces of the placenta [3740]. Protective immunity against the pregnancy-related placental malaria is acquired over multiple pregnancies and thus, VAR2CSA can be a major target for the development of vaccine against placental malaria $[39,41]$. In this study, VAR2CSA homologs are detected only in P. reichenowi and P. gaboni, but not in other primate and rodent malaria (Fig. 3). Currently, it is uncertain whether the EMP1 protein plays the adhesive role, similar to the VAR2CSA protein of $P$. falciparum, in rodent malaria. Nevertheless, the functional analog(s) of VAR2CSA is likely to be expressed in rodent malaria, considering many features that are commonly observed in the murine and human placental malaria [42]. Screening of the functional analog(s) in murine system is necessary to use the rodent malaria as an effective experimental model of human placental malaria.

To date, numerous recombinant proteins and crude bloodstage antigens of plasmodial species have been applied in the experimental investigations of protective immunity against malaria using model animals $[14,26,43]$. The target proteins mixed with various adjuvants were injected into experimental animals in a large amount to invoke potent immune response in these studies [14]. However, the ability to stimulate host immune system for the generation of specific protective antibodies appears to be quite different among vaccine candidates in practical patients [26]. Therefore, there have been more recent efforts to overcome/compensate the clinical limitation of previously established vaccine candidates by selecting immunogenic antigens against naturally acquired antibodies [4345]. Several protein bands expressed in the blood-stage P. yoelii $17 \mathrm{XNL}$ were highly reactive with specific antibodies in experimental mice sera recovered from the primary challenge of the parasite. The low or negligible parasitemia observed in these 
mice might result from the neutralizing action of the protective antibodies (Fig. 4). In addition to the generation of recombinant proteins characterized in this study for protective test, experiments for identification and cloning of the immunogenic antigens shown in Fig. 4D via mass spectrometry and following Mascot analyses are under preparations. Vaccination effects of the 17XNL proteins will also be further examined in mice challenged with the lethal P. yoelii 17X strain. The results obtained by the near-future studies might provide the necessary information and means to characterize additional vaccine candidate antigens, selected solely on their ability to produce the protective antibodies.

\section{ACKNOWLEDGMENT}

This research was supported by a grant from the Korea Health Technology R\&D Project through the Korea Health Industry Development Institute (KHIDI), funded by the Ministry of Health and Welfare, the Republic of Korea (grant no. HI15C3050).

\section{CONFLICT OF INTEREST}

We have no conflict of interest related to this work.

\section{REFERENCE}

1. White NJ. Plasmodium knowlesi: the fifth human malaria parasite. Clin Infect Dis 2008; 46: 172-173.

2. Singh B, Daneshvar C. Human infections and detection of Plasmodium knowlesi. Clin Microbiol Rev 2013; 26: 165-184.

3. World Health Organization. World Malaria Report: 2016. Geneva, Switzerland, World Health Organization.

4. Cui L, Mharakurwa S, Ndiaye D, Rathod PK, Rosenthal PJ. Antimalarial drug resistance: literature review and activities and findings of the ICEMR network. Am J Trop Med Hyg. 2015; 93(3 Suppl): 57-68.

5. Richards JS, Beeson JG. The future for blood-stage vaccines against malaria. Immunol Cell Biol 2009; 87: 377-390.

6. Nunes JK, Woods C, Carter T, Raphael T, Morin MJ, Diallo D, Leboulleux D, Jain S, Loucq C, Kaslow DC, Brikett AJ. Development of a transmission-blocking malaria vaccine: progress, challenges, and the path forward. Vaccine 2014; 32: 5531-5539.

7. Ouattara A, Laurens MB. Vaccines against malaria. Clin Infect Dis 2015; 60: 930-936.

8. Craig AG, Grau GE, Janes C, Kazura JW, Milner D, Barnwell JW, Turner G, Langhorne J; participants of the Hinxton Retreat meeting on Animal Models fro Research on Severe Malaria. The role of animal models for research on severe malaria. PLoS Pathog 2012; 8: e1002401.

9. Otto TD, Böhme U, Jackson AP, Hunt M, Franke-Fayard B, Hoeijmakers WA, Religa AA, Robertson L, Sanders M, Ogun SA, Cunningham D, Erhart A, Billker O, Khan SM, Stunnenberg HG, Langhorne J, Holder AA, Waters AP, Newbold CI, Pain A, Berriman $\mathrm{M}$, Janse CJ. A comprehensive evaluation of rodent malaria parasite genomes and gene expression. BMC Biol 2014; 12: 86 .

10. Lamb TJ, Brown DE, Potocnik AJ, Langhorne J. Insights into the immunopathogenesis of malaria using mouse models. Expert Rev Mol Med 2006; 8: 1-22.

11. Kappe SH, Vaughan AM, Boddey JA, Cowman AF. That was then but this is now: malaria research in the time of an eradication agenda. Science 2010; 328: 862-866.

12. Prudêncio M, Mota MM, Mendes AM. A toolbox to study liver stage malaria. Trends Parasitol 2011; 27: 565-574.

13. Ogun SA, Tewari R, Otto TD, Howell SA, Knuepfer E, Cunningham DA, Xu Z, Pain A, Holder AA. Targeted disruption of py235ebp-1: invasion of erythrocytes by Plasmodium yoelii using an alternative Py235 erythrocyte binding protein. PLoS Pathog 2011; 7: e1001288.

14. Burns JM Jr, Dunn PD, Russo DM. Protective immunity against Plasmodium yoelii malaria induced by immunization with particulate blood-stage antigens. Infect Immun 1997; 65: 3138-3145.

15. Wykes MN, Zhou YH, Liu XQ, Good MF. Plasmodium yoelii can ablate vaccine-induced long-term protection in mice. J Immunol 2005; 175: 2510-2516.

16. Wipasa J, Xu H, Liu X, Hirunpetcharat C, Stowers A, Good MF. Effect of Plasmodium yoelii exposure on vaccination with the 19-kilodalton carboxyl terminus of merozoite surface protein 1 and vice versa and implications for the application of a human malaria vaccine. Infect Immun 2009; 77: 817-824.

17. Hoffman EJ, Weidanz WP, Long CA. Susceptibility of CXB recombinant inbred mice to murine plasmodia. Infect Immun 1984; 43: 981-985.

18. Janse CJ, Ramesar J, Waters AP. High-efficiency transfection and drug selection of genetically transformed blood stages of the rodent malaria parasite Plasmodium berghei. Nat Protoc 2006; 1: 346-356.

19. Mount SM. A catalogue of splice junction sequences. Nucleic Acids Res 1982; 10: 459-472.

20. Farley PJ, Srivastava R, Long CA. Sequence of the gene encoding the N-terminal portion of the Plasmodium yoelii yoelii 17XL merozoite surface protein-1 (MSP-1). Gene 1994; 151: 335-336.

21. Jennings GJ, Toebe CS, van Belkum A, Wiser MF. The complete sequence of Plasmodium berghei merozoite surface protein-1 and its inter- and intra-species variability. Mol Biochem Parasitol 1998; 93: 43-55.

22. Pacheco MA, Battistuzzi FU, Junge RE, Cornejo OE, Williams CV, Landau I, Rabetafika L, Snounou G, Jones-Engel L, Escalante AA. Timing the origin of human malarias: the lemur puzzle. BMC Evol Biol 2011; 11: 299.

23. de la Cruz VF, Lal AA, McCutchan TF. Variation among circum- 
sporozoite protein genes from rodent malarias. Mol Biochem Parasitol 1988; 28: 31-38.

24. Franke ED, Sette A, Sacci J Jr, Southwood S, Corradin G, Hoffman SL. A subdominant CD8(+) cytotoxic T lymphocyte (CTL) epitope from the Plasmodium yoelii circumsporozoite protein induces CTLs that eliminate infected hepatocytes from culture. Infect Immun 2000; 68: 3403-3411.

25. Cohen S, McGregor IA, Carrington S. Gamma-globulin and acquired immunity to human malaria. Nature 1961; 192: 733737.

26. Fowkes FJ, Richards JS, Simpson JA, Beeson JG. The relationship between anti-merozoite antibodies and incidence of Plasmodium falciparum malaria: a systematic review and meta-analysis. PLoS Med 2010; 7: e1000218.

27. Diggs CL, Ballou WR, Miller LH. The major merozoite surface protein as a malaria vaccine target. Parasitol Today 1993; $9: 300-$ 302.

28. Holder AA. The carboxy-terminus of merozoite surface protein 1: structure, specific antibodies and immunity to malaria. Parasitology 2009; 136: 1445-1456.

29. Versiani FG, Almeida ME, Mariuba LA, Orlandi PP, Nogueira PA. N-terminal Plasmodium vivax merozoite surface protein-1, a potential subunit for malaria vivax vaccine. Clin Dev Immunol 2013; 2013: 965841.

30. Curd RD, Birdsall B, Kadekoppala M, Ogun SA, Kelly G, Holder AA. The structure of Plasmodium yoelii merozoite surface protein 119 , antibody specificity and implications for malaria vaccine design. Open Biol 2014; 4: 130091.

31. Aikawa M, Yoshida N, Nussenzweig RS, Nussenzweig V. The protective antigen of malarial sporozoites (Plasmodium berghei) is a differentiation antigen. J Immunol 1981; 126: 2494-2495.

32. Zavala F, Cochrane AH, Nardin EH, Nussenzweig RS, Nussenzweig V. Circumsporozoite proteins of malaria parasites contain a single immunodominant region with two or more identical epitopes. J Exp Med 1983; 157: 1947-1957.

33. Eichinger DJ, Arnot DE, Tam JP, Nussenzweig V, Enea V. Circumsporozoite protein of Plasmodium berghei: gene cloning and identification of the immunodominant epitopes. Mol Cell Biol 1986; 6: 3965-3972.

34. Espinosa DA, Gutierrez GM, Rojas-López M, Noe AR, Shi L, Tse SW, Sinnis P, Zavala F. Proteolytic cleavage of the Plasmodium falciparum circumsporozoite protein is a target of protective antibodies. J Infect Dis 2015; 212: 1111-1119.

35. David PH, Hommel M, Miller LH, Udeinya IJ, Oligino LD. Para- site sequestration in Plasmodium falciparum malaria: spleen and antibody modulation of cytoadherence of infected erythrocytes. Proc Natl Acad Sci USA 1983; 80: 5075-5079.

36. Yamada S, Sugahara K. Potential therapeutic application of chondroitin sulfate/dermatan sulfate. Curr Drug Discov Technol 2008; 5: 289-301.

37. Smith JD, Chitnis CE, Craig AG, Roberts DJ, Hudson-Taylor DE, Peterson DS, Pinches R, Newbold CI, Miller LH. Switches in expression of Plasmodium falciparum var genes correlate with changes in antigenic and cytoadherent phenotypes of infected erythrocytes. Cell 1995; 82: 101-110.

38. Achur RN, Valiyaveettil M, Alkhalil A, Ockenhouse CF, Gowda DC. Characterization of proteoglycans of human placenta and identification of unique chondroitin sulfate proteoglycans of the intervillous spaces that mediate the adherence of Plasmodium falciparum-infected erythrocytes to the placenta. J Biol Chem 2000; 275: 40344-40356.

39. Salanti A, Staalsoe T, Lavstsen T, Jensen AT, Sowa MP, Arnot DE, Hviid L, Theander TG. Selective up-regulation of a single distinctly structured var gene in chondroitin sulfate A-adhering Plasmodium falciparum involved in pregnancy-associated malaria. Mol Microbiol 2003; 49: 179-191.

40. Clausen TM, Christoffersen S, Dahlbäck M, Langkilde AE, Jensen KE, Resende M, Agerbæk M, Andersen D, Berisha B, Ditlev SB, Pinto VV, Nielsen MA, Theander TG, Larsen S, Salanti A. Structural and functional insight into how the Plasmodium falciparum VAR2CSA protein mediates binding to chondroitin sulfate $\mathrm{A}$ in placental malaria. J Biol Chem 2012; 287: 23332-23345.

41. Brabin BJ. An analysis of malaria in pregnancy in Africa. Bull World Health Organ 1983; 61: 1005-1016.

42. Hviid, L, Marinho CR, Staalsoe T, Penha-Gonçalves C. Of mice and women: rodent models of placental malaria. Trends Parasitol 2010; 26: 412-419.

43. Chia WN, Goh YS, Rénia L. Novel approaches to identify protective malaria vaccine candidates. Front Microbiol 2014; 5: 586.

44. Davies DH, Duffy P, Bodmer JL, Felgner PL, Doolan DL. Large screen approaches to identify novel malaria vaccine candidates. Vaccine 2015; 33: 7496-7505.

45. França CT, Hostetler JB, Sharma S, White MT, Lin E, Kiniboro B, Waltmann A, Darcy AW, Li Wai Suen CS, Siba P, King CL, Rayner JC, Fairhurst RM, Mueller I. An antibody screen of a Plasmodium vivax antigen library identifies novel merozoite proteins associated with clinical protection. PLoS Negl Trop Dis 2016; 10: e0004639. 
Supplementary Table 1. Primers used in the amplification of Plasmodium yoelii MSP1 and CSP gene segments for the generation of recombinant proteins

\begin{tabular}{|c|c|c|c|c|}
\hline Protein $^{\mathrm{a}}$ & Oligo name & Sequence $\left(5^{\prime} \rightarrow 3^{\prime}\right)^{b}$ & Amplified base positions & Recombinant protein \\
\hline \multirow[t]{2}{*}{ rPyMSP1-1 } & PyMSP1Nt1-F & CCGGATCC ${ }^{1}$ ACAATTGAAGTTTATAATG & $61-1065$ & \\
\hline & PyMSP1Nt1-R & GCGCGGCCGC²GTTAAGTTGGAACGGGTTGC & & \\
\hline \multirow[t]{2}{*}{ rPyMSP1-2 } & PyMSP1Nt2-F & CCGGATCC ${ }^{1}$ CTTACATTAGAAGAAAAACAG & $1066-2103$ & \\
\hline & PyMSP1Nt2-R & GGGCGGCCGC ${ }^{2}$ GTTATाTCTाCGATACTाC & & \\
\hline \multirow[t]{2}{*}{ rPyMSP1-3 } & PyMSP1Mt1-F & CAGGATCC $^{1}$ ATTGCTAAAGAGAAG & $2104-2490$ & \\
\hline & PyMSP1Mt1-R & GCGCGGCCGC ${ }^{2}$ GTTATGATGCTTCTGTTGAG & & \\
\hline \multirow[t]{2}{*}{ rPyMSP1-4 } & PyMSP1Mt2-F & CAGGATCC ${ }^{1}$ TCATCAGCACCTGGCGAAGG & $2941-3678$ & Yes \\
\hline & PyMSP1Mt2-R & GGGCGGCCGC²GTTAAGAAGTAAAATATTTAG & & \\
\hline \multirow[t]{2}{*}{ rPyMSP1-5 } & PyMSP1Ct1-F & CGGGATCC ${ }^{1}$ ATGGAAGCTGTTCCTTTAAAAAC & $3679-4344$ & Yes \\
\hline & PyMSP1Ct1-R & GCGCGGCCGC²GTATAATCCATTTGTCATG & & \\
\hline \multirow[t]{2}{*}{ rPyMSP1-6 } & PyMSP1Ct2-F & CGGGATCC ${ }^{1}$ AATAATAAATCACAAAAAAG & $4345-5331$ & \\
\hline & PyMSP1Ct2-R & GGGCGGCCGC²GTTAAAATATATTAAATAC & & \\
\hline \multirow[t]{2}{*}{ rPyMSP1-7 } & PyMSP1Ct2-C19-F & CGGGATCC ${ }^{1}$ ATGGAAGCTGTTCCTITAAAAAC & $4345-5223$ & \\
\hline & PyMSP1Ct2-C19-R & GCGCGGCCGC ${ }^{2}$ GTTATTACATGTACATATAATT & & \\
\hline \multirow[t]{2}{*}{ rPyMSP1-8 } & PyMSP1C19-F & CCGGATCC ${ }^{1}$ CCGAACCAACCCCTAATGC & $5224-5331$ & Yes \\
\hline & PyMSP1C19-R & GGGCGGCCGC ${ }^{2}$ GTTAAAATATATTAAATAC & & \\
\hline \multirow[t]{2}{*}{ rPyCSP-1 } & PyCSPNterm-F & CGGGATCC $^{1}$ AATAAAAGTGTCCAAGCCC & $73-684$ & Yes \\
\hline & PyCSPNterm-R & GCGCGGCCGC² ${ }^{2}$ TTATGGTGCTCCTGGTCCTTG & & \\
\hline \multirow[t]{2}{*}{ rPyCSP-2 } & PyCSPCterm-F & CGGGATCC ${ }^{1}$ CCACAAGAACCACCCCAAC & $862-1284$ & Yes \\
\hline & PyCSPCterm-R & GCCTCGAG ${ }^{3}$ GTTAATTAAAGAATACTAATAC & & \\
\hline
\end{tabular}

${ }^{a}$ Amplified gene segments were cloned into pET-28a plasmid except for Clone 8, which was inserted into pGEX-6p-1.

bUnderlined sequences are recognition sites for BamHI (1), Notl (2) and Xhol (3), respectively. 


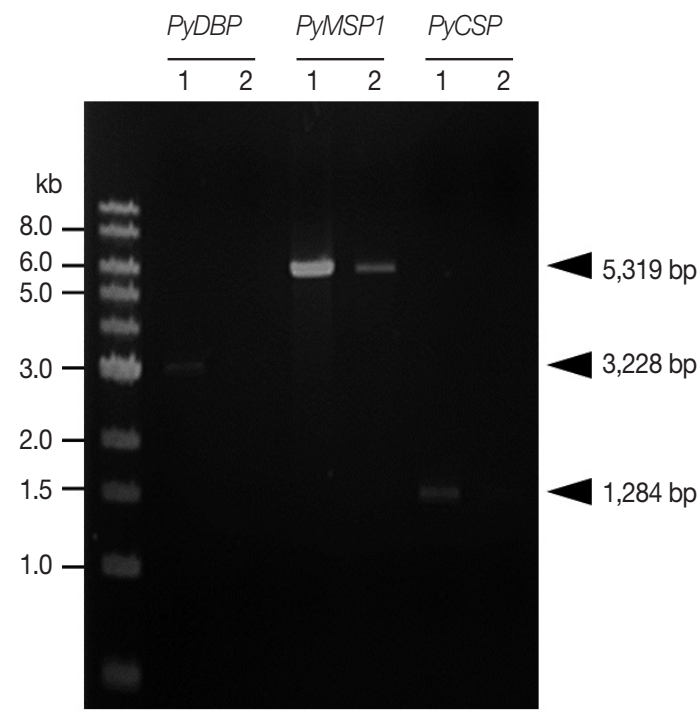

Supplementary Fig. 1. Amplification of Plasmodium yoelii genes. The $P$. yoelii genes encoding Duffy-binding protein (PyDBP), merozoite surface protein 1 (PyMSP1), and circumsporozoite protein (PyCSP) were amplified from genomic DNA of the parasite by a long-range PCR using respective gene-specific primers. The PCR products were electrophoresed on a $0.8 \%$-agarose gel and visualized by ethidium bromide staining. Lane 1, products of secondary PCR (cycling numbers =25); lane 2, products of first PCR (cycling numbers $=35$ ). 